\title{
Optimization of risk criteria for road tunnels
}

\author{
M. Holický \\ Klokner Institute, CTU in Prague, Czech Republic
}

\begin{abstract}
Available documents concerning risk management and assessment of road tunnels provide almost no guidance on risk criteria. Three different types of risk are discussed in the submitted paper: individual risk $R_{\text {ind }}$ of a person related to specific operational conditions, expected risk $R_{\exp }$ expressed in terms of number of fatalities per tunnel and year and societal risk expressed in terms of cumulative frequency $F=\mathrm{P}\left(R_{m} \geq N\right)$, presented commonly as $F-N$ curve, per tunnel (or $1 \mathrm{~km}$ of a tunnel) and year. Recommendations for quantitative risk criteria are derived from commonly accepted safety requirements. The ALARP concept is generally accepted for the specification of the lower and upper bounds of relevant risk indicators. The lower and upper bounds of an individual risk are within the interval from $10^{-3}$ to $10^{-6}$ per year and tunnel. The expected risk is to be anticipated around one fatality per tunnel and year. The societal risk is commonly compared with the $F-N$ curve given by a power function $A N^{-k}$, where the cumulative frequency $A$ is within the interval from 0,0001 to 0,1 and the parameter $k$ within the interval from 1 to 2 . Further it is shown that expected risk $R_{\exp }$ can be used to minimize the total consequences including consequences of unfavourable events and costs of safety measures (for example escape routes). It appears that the assumed life time and discount rate may significantly affect the total consequences and the optimum arrangements of the road tunnels.

Keywords: risk, criteria, road, tunnels, optimization.
\end{abstract}

\section{Introduction}

Road tunnels represent complex technical systems that may be exposed to hazard situations leading to serious consequences. Risk criteria for road tunnels are therefore becoming a more and more important issue all over the word. The increasing interest in tunnel safety caused by recent tragic events in several European road tunnels resulted in an effort to take consequences of unfavorable 
events into consideration in design of new and assessment of existing tunnels. In case of trans-European road network, these requirements are specified in the Directives of the European Parliament and of the Council 2004/54/ES [4]. The directive also includes guidance on risk management, assessment and risk control.

However, no quantitative recommendations are provided in official European documents on types of risk and their acceptance criteria. The submitted paper provides information on some national (CAN/CSA [1], NS 5814 [14]) and international standards (ISO [10-13]) and literature (Stewart and Melchers [17], Melchers [9]) and offer recommendations on risk criteria. It appears that three different types of risk are often considered in case of road tunnels (Vrouwenvelder et al. [19], Trbojevic [18], Brussaard et al. [1], Knoflacher Pfaffenbichler [8], Ruffin et al. [15]):

- $\quad$ individual risk $R_{\text {ind }}$ of a person related to specific operational conditions,

- $\quad$ expected risk $R_{\text {exp }}$ expressed in terms of number of fatalities per tunnel and year,

- $\quad$ societal risk expressed in terms of cumulative frequency $F=\mathrm{P}\left(R_{m} \geq N\right)$ that number of fatalities exceeds a given $N$ per tunnel or $1 \mathrm{~km}$ of a tunnel and year (called $F-N$ curve).

It is also indicated that the expected risk $R_{\exp }$ may be used to analyse the overall tunnel utility and to apply probabilistic methods of risk optimizations. The optimum expected risk may be then specified taking into account a given design working life of a tunnel (100 years) and discount rate (around $3 \%$ ).

\section{ALARP concept}

The target values in criteria are usually given by the lower and upper bounds of area denoted by the abbreviation ALARP (As Low As Reasonably Practicable). The ALARP concept is shown by Trbojevic [18].

If the risk indicator is above the upper bound, the risk level is considered as unacceptable. If the risk indicator is below the lower bound, then the risk level is considered as acceptable. If the risk indicator is between the limits, then the risk level should be reduced depending on technical conditions and costs of relevant measures.

\section{The individual risk}

Three types of consequences are considered in case of road tunnels: individual risk, expected risk and societal risk. The individual risk is the probability that a particular person (a member of operating staff checking regularly tunnel conditions) loses his life. Assuming that during his service hazard situations $H_{i}$ may cause a set of events $E_{i j}$ (for example fully developed fire, explosion). Let $p_{i j}$ denote the probabilities that the operator loses his life. Then the total individual risk of that person can be estimated using a formal expression

$$
p_{\text {ind }}=\sum_{i j} p_{i j} \mathrm{P}\left(E_{i j} \mid H_{i}\right) \mathrm{P}\left(H_{i}\right)
$$


The acceptable lower limit for the individual risk $R_{\text {ind,t }}$ is usually based on annual probability $10^{-6}$ (ISO 2394 [10], Trbojevic [18]), which is commonly accepted for the loss of life due to structural failure or due to electric power and radiation (Trbojevic [18]). This lower bound is accepted without other consideration concerning possible risk reduction (see Figure 1).

More complicated seems to be the specification of the upper bound for individual risk that should not be exceeded (see Figure 1). In that case various semi probabilistic and empirical estimations are applied. For example, in most industrial areas the annual probability of fatality $10^{-3}$ is accepted (Trbojevic [18]). This is, however, only a heuristic assessment based on experience and not on any rational analysis. The lower bound $10^{-3}$ also well corresponds to annual fatalities observed in construction. However, in other industrial areas the annual probability may be lower.

The individual risk $p_{\text {ind }}$ may be compared with the target value $p_{\text {ind, }}$ provided in ISO 2394 [10], where an informative value $10^{-6}$ per one year is indicated. However, in industrial conditions a greater value $10^{-3}$ is commonly accepted. Thus the criterion for the individual risk may be expressed as a simple inequality

$$
p_{\text {ind }} \leq p_{\text {ind, } \mathrm{t}} \approx 10^{-6} \text { to } 10^{-3} \text { per year and tunnel }
$$

As already indicated in the case of road tunnels, the individual risk may concern persons performing regular activity in a tunnel, for example the maintenance of inspection. The individual risk should be therefore distinguished from expected number of fatalities that is a general characteristic of a tunnel based on an expected traffic density and tunnel properties.

\section{The expected risk}

The expected risk $R_{\text {exp }}$ describes number of casualties resulting from normal operational conditions of a tunnel. Formally it can be estimated similarly as the individual risk using an expression

$$
R_{\exp }=\sum_{i j} C_{i j} \mathrm{P}\left(E_{i j} \mid H_{i}\right) \mathrm{P}\left(H_{i}\right)
$$

Here symbol $C_{i j}$ denotes consequences corresponding to the event $E_{i j}$, for example number of casualties in case the event $E_{i j}$ occurs. No recommendations are available for the target value of expected risk $R_{\text {exp, }, \text {. However, comparing }}$ symbolic expressions (1) and (2) it is obvious that $R_{\text {exp.t }}$ could be expected around one casualty per year and tunnel. Thus the criterion for the individual risk may be expressed as a simple inequality

$$
R_{\text {exp }} \leq R_{\text {exp,t }} \text { per year and tunnel }
$$

It does not reflect a particular person, but all passengers entering the tunnel. A very rough estimate may be obtained from the bounds $p_{\text {ind, }}$ for the individual risk 
and equations (2) and (4). Assuming a very approximate ratio between the average values of $C_{\mathrm{ij}}$ and $p_{\mathrm{ij}}$ around $10^{3}$, then the upper bound for the expected risk could be around unity per year and tunnel.

It should be noted that the expected risk can be used in optimization of tunnel arrangement, for example when the number of escape routes is considered as a decisive parameter of a tunnel (Holický [6], Holický and Šajtar [5], Holický et al. [7]). The optimum number of escape routes and a corresponding level of expected risk may be derived from the requirement for the minimum of the total costs.

\section{The societal risk}

The societal risk is commonly expressed as cumulative probability (cumulative frequency) that the number of casualties is equal or greater than a number $N$

$$
F=\mathrm{P}\left(R_{m} \geq N\right)=\sum_{l \geq m} f_{l}
$$

Here the indices $m$ and $l$ are used to denote particular number $N$ considered in the analysis. Cumulative frequency $F$ is commonly compared with a target value $F_{\mathrm{t}}=A N^{-k}$. The power function $A N^{-k}$ known as $(F-N)$ curve is used in several technical areas (Vrouwenvelder et al. [19], Trbojevic [18]). Thus the criterion for the expected risk may be expressed as an inequality

$$
F \leq F_{\mathrm{t}}=A N^{-k}
$$

The criterion of societal risk (6) is usually shown in a graph with logarithmic scale for both the number $N$ (within the interval from 1 to 1000) on horizontal axes and the cumulative frequency $F$ (cumulative probability $\mathrm{P}\left(R_{m}>N\right)$ ) on the vertical axes (within the interval from $10^{-8}$ to 1 ). Then the frequency curve is represented by a line having the slope $-k$ ( $k$ is commonly given by the values within the interval from 1 to 2 (Trbojevic [18]). The parameters $A$ and $k$ specify the shape of the curve. Note that the parameter $A$ is equal to the cumulative frequency $F=\mathrm{P}\left(R_{m} \geq 1\right)$, e.g. cumulative probability for $N=1$.

\section{Acceptable levels of societal risks}

Acceptable level of societal risk is described by equation (6) (Vrouwenvelder et al. [19]). Figure 1 shows criteria most often used in case of road tunnels (Vrouwenvelder et al. [19], Trbojevic [18]), that differ by parameters $A$ and $k$ of the power function $A N^{-k}$ :

(1) moderate upper bound: $A=0,1$ and $k=1$

(2) severe upper bound: $A=0,01$ and $k=1$

(3) middle level: $A=0,1$ and $k=2$ (Westerschelde)

(4) moderate lower bound: $A=0,01$ and $k=2$

(5) severe lower bound: $A=0,0001$ and $k=1$ 
All the above mentioned criteria are shown in Figure 1. The moderate upper bound (1) is applied for example in UK (as indicated by Vrouwenvelder et al. [19]). The severe upper bound (2) $A=0,01$ and $k=1$ is recommended by Trbojevic V. M. 2003. The middle level (3) for $A=0,1$ and $k=2$ was accepted for Westerschelde tunnel in the Netherlands (Worm and Hoeskma [22]). The moderate lower bound, $A=0,01$ and $k=2$ and severe lower bound (5), $A=$ 0,0001 and $k=1$ is considered by Knoflacher and Pfaffenbichler [8] and Trbojevic [18].

Note that if the parameter $k=1$, then it follows from criterion (6) that the target frequency $F_{\mathrm{t}}$ (requirement for the frequency $F=\mathrm{P}\left(R_{m} \geq N\right)$ of equal or more casualties than $N$ is equal to $A / N$. Thus in that case, for a given total cumulative frequency $A$ the required target frequency $F_{\mathrm{t}}$ is an inversely proportion to the number of casualties $N$.

It appears that the upper bound (2) and the lower bound (5) $(\mathrm{k}=1)$, recommended in a recent study by Trbojevic 2003, represent a sufficiently broad area ALARP. The acceptable cumulative probability $P\left(R_{m}>N\right)$ of one or more fatalities occurring is within the range from 0,0001 to 0,10 . However, the lower bound (5) seems to be rather severe and difficult to achieve.

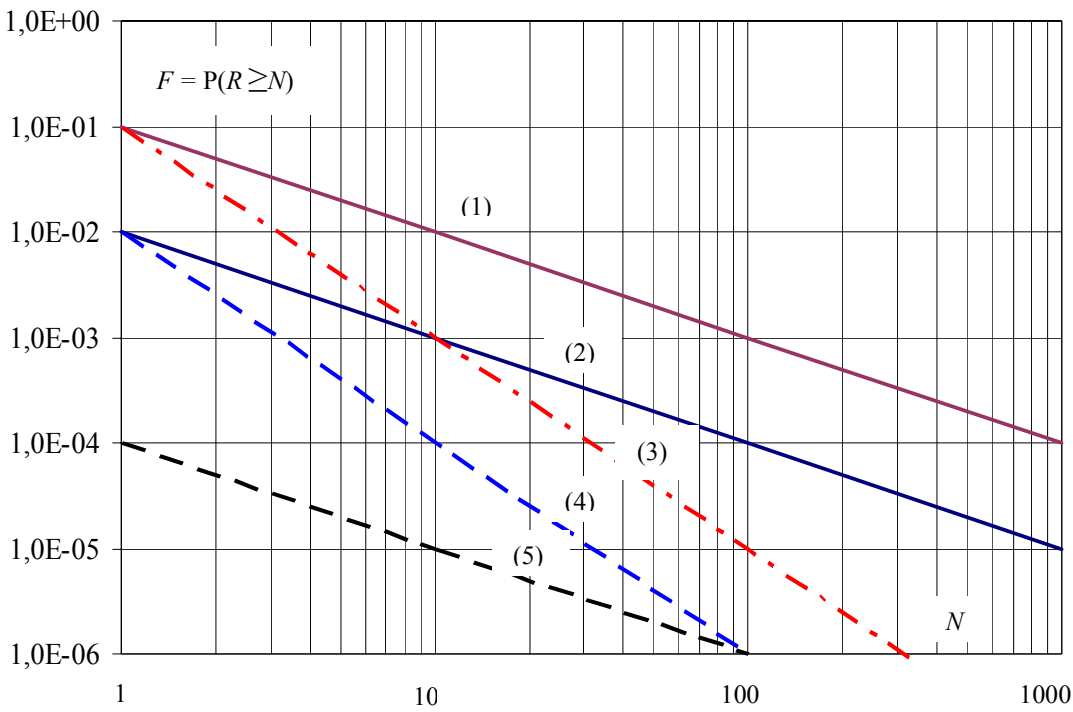

Figure 1: $\quad$ Societal risk bounds.

\section{Risk optimization}

The total consequences $C_{\text {tot }}(k, p, n)$ relevant to the construction and performance of the tunnel are generally expressed as a function of the decisive parameter $k$ (for example of the number $k$ of escape routes), discount rate $p$ (commonly about $p \approx 0,03$ ) and life time $n$ (commonly $n=100$ let). The decisive parameter $k$ 
usually represents a one-dimensional or multidimensional quantity significantly affecting tunnel safety.

The fundamental model of the total consequences may be written as a sum of partial consequences as (Holický and Šajtar [5], Holický et al. [7]).

$$
C_{\text {tot }}(k, p, n)=R(k, p, n)+C_{0}+\Delta C(k)
$$

In equation (7) $R(k, p, n)$ denotes expected societal risk that is dependent on the parameter $k$, discount rate $p$ and life time $n$. $C_{0}$ denotes the initial construction cost independent of $k$, and $\Delta C(k)$ additional expenses dependent on $k$. Equation (7) represents, however, only a simplified model that does not reflect all possible expenses including economic consequences of different unfavorable events and maintenance costs. The societal risk $R(k, p, n)$ may be estimated using the following formulae

$$
R(k, p, n)=N(k) R_{1} Q(p, n), \quad Q(p, n)=\frac{1-1 /(1+p)^{n}}{1-1 /(1+p)}
$$

In equation (8) $N(k)$ denotes number of expected fatalities per one year (dependent on $k$ ), $R_{1}$ denotes acceptable expenses for averting one fatality, and $p$ the discount rate (commonly within the interval from 0 to $5 \%$ ). The quotient $q$ of the geometric row is given by the fraction $q=1 /(1+p)$. The discount coefficient $Q(p, n)$ makes it possible to express the actual expenses $Z_{1}$ during a considered life time $n$ in current cost considered in (7). In other words, expenses $Z_{1}$ in a year $i$ correspond to the current $\operatorname{cost} R_{1} q^{\mathrm{i}}$. The sum of the expenses during $n$ years is given by the coefficient $Q(p, n)$.

The total consequences given by equation (7) may be in some cases simplified to a dimensionless standardized form and the whole procedure of optimization may be generalized. Consider as an example the optimization of the number $k$ of escape routes. It is assumed that involved additional costs $\Delta C(k)$ due to $k$ may be expressed as the product $k C_{1}$, where $C_{1}$ denotes the cost of one escape route. If $C_{1}$ is approximately equal to $R_{1}$ (assumed also by Vrouwenvelder and Krom [20]), equation (7) becomes

$$
C_{\text {tot }}(k, p, n)=N(k) C_{1} Q(p, n)+C_{0}+k C_{1}
$$

This function can be standardized as follows

$$
\kappa(k, p, n)=\frac{C_{t o t}(k, p, n)-C_{0}}{C_{1}}=N(k) Q(p, n)+k
$$

Figure 2 shows the variation of the total consequences $\kappa(k, p, n)$ with the number of escape routes $k$ and discount rate $p$ assuming again the expected life $n$ $=100$ years.

Both variables $C_{\text {tot }}(k, p, n)$ and $\kappa(k, p, n)$ are mutually dependent and have the extremes (if exist) for the same number of escape routes $k$. A necessary condition for the extremes following from (10) is 


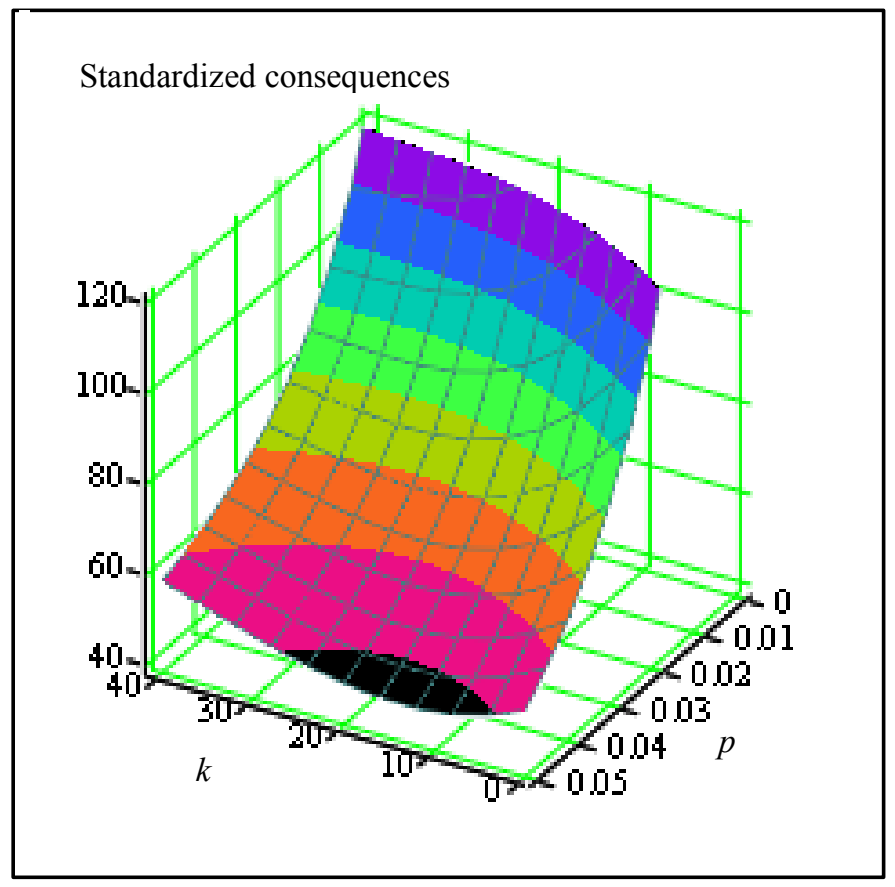

Figure 2: Variation of the standardized total consequences $\kappa(k, p, n)$ with the number of escape routes $\mathrm{k}$ and discount rates $p$ for the life time $n=$ 100 years.

$$
\frac{\partial N(k)}{\partial k}=-\frac{1}{Q(p, n)}=-\frac{1-1 /(1+p)}{1-1 /(1+p)^{n}}
$$

An advantage of standardized consequences is the fact that it is independent of $C_{0}$ and $C_{1}$. It is only assumed that $C_{1} \approx Z_{1}$ is a time invariant unit of the total consequences. However, this assumption should be considered as an approximation only and should be revised in a particular tunnel. A more general study when the ratio of values $C_{1}$ and $Z_{1}$ is considered as an additional parameter is provided by Holický [6]. Then expenses, which a society is able to afford for averting one fatality (societal compensation cost), must be taken into account explicitly. The concept of life quality index - LQI (Rackwitz [16]) or Value for Averted Fatality - VAF (EN 1991-1-7 [4]) may be then applied.

\section{Conclusions}

Risk criteria for road tunnels are becoming a more and more important issue all over the word. Three types of risk are commonly applied in the safety assessment of road tunnels: 
- the individual risk per year and tunnel,

- the expected risk per year and tunnel,

- $\quad$ the societal risk ( $F$ - $N$ curve) per year and tunnel.

No specific requirements are recommended in available international documents. The ALARP concept is generally accepted for the specification of the lower and upper bounds of relevant risk indicators. The lower and upper bounds of an individual risk are within the interval from $10^{-3}$ to $10^{-6}$ per year and tunnel. The expected risk is to be anticipated around one fatality per tunnel and year. The societal risk is commonly compared with the $F-N$ curve given by a power function $A N^{-k}$, where the cumulative frequency $A$ is within the interval from 0,0001 to 0,1 and the parameter $k$ within the interval from 1 to 2 .

The expected risk may be used for probabilistic risk optimization based on the minimization of the total cost. The risk optimization may provide valuable background information for a rational decision concerning effective safety measures applied to road tunnels. However, as tunnels are commonly designed for a long life time (100 years), discount rate must be taken into account. It appears that the assumed life time and discount rate may affect the total consequences and the optimum arrangements of the tunnels more significantly than the number of escape routes. In general, expenses, which a society is able to afford for averting one fatality (VPF - value for prevented fatality or societal compensation cost), must be also taken into account.

The following conclusions may be drawn from the study of the probabilistic risk optimization of road tunnels using Bayesian networks:

- The optimum number of escape routes may be specified from the requirement for the minimum total consequences covering both the societal and economic aspects.

- The optimum number of escape routes depends generally on the discount rate, required life time and the ratio between the cost for one escape route and acceptable expenses, which a society is able to afford for averting one fatality (societal compensation cost).

- The total consequences are primarily affected by the discount rate and less significantly by the assumed life time, cost ratio and the number of escape routes.

A correct specification of discount rate and required life time is essential for making proper decisions. Further investigations of input data concerning conditional probabilities describing individual hazard scenarios and models for their societal and economic consequences are needed.

\section{Acknowledgement}

This study is a part of the project GAČR 103/06/1521 "Reliability and Risk Assessment of Structures in Extreme Conditions" supported by the Grant Agency of the Czech Republic. 


\section{References}

[1] Brussaard L.A., Kruiskamp M.M. and Oude Essink M.P. (2004) The Dutch model for the quantitative risk analysis of road tunnels. ESREL 2004, Berlin, June 2004.

[2] CAN/CSA-Q634-91 (1991) Risk analysis requirements and guidelines. 1991.

[3] Directive (2004) 2004/54/EC of the European Parliament and of the Council of 29 April 2004 on minimum safety requirements for tunnels in the trans-European road network. Official Journal of the European Union L 201/56 of 7 June 2004.

[4] EN 1991-1-7 (2006) Eurocode 1 - Actions on structures - Part 1-7: General actions - Accidental actions, CEN, 2006.

[5] Holický M., Šajtar L. (2006) Probabilistic risk assessment and optimization of road tunnels. In Safety and Reliability for managing Risk Guedes Soares and Zio (eds), Tailor and Francis Group, London, 2006, pp. 297-304.

[6] Holický M. (2006) Risk analysis and optimization of road tunnels. In.: Risk Analysis V: Simulation and Hazard Mitigation. WIT press 2006, Southampton, Boston. pp. 57-66.

[7] Holický M., Šajtar L. and Smolík J. (2007) Optimisation of Road Tunnel Safety Based on Risk Analysis, World Tunnel Congress 2007, Prague, to be published.

[8] Knoflacher H. and P.C. Pfaffenbichler (2004) A comparative risk analysis for selected Austrian tunnels. 2nd International Conference Tunnel Safety and Ventilation, Graz, 2004.

[9] Melchers R.E. (1999) Structural reliability analysis and prediction. John Wiley \& Sons, Chichester, 1999, 437 p.

[10] ISO 2394 (1998) General principles on reliability for structures. 1998.

[11] ISO/IEC Guide 73 (2002) Risk management - Vocabulary - Guidelines for use in standards.

[12] ISO/IEC Guide 51 (1999), Safety aspects - Guidelines for their inclusion in standards.

[13] ISO 9000 (2000) Quality management systems - Fundamentals and vocabulary.

[14] NS 5814 (1991) Requirements for risk analysis. 1991.

[15] Ruffin E., P. Cassini P. and H. Knoflacher (2005) Transport of hazardous goods. See chapter 17 of Beard A and Carvel R (2005). The Handbook of Tunnel Fire Safety. Thomas Telford Ltd, London, 2005.

[16] Rackwitz R (2005) R. The philosophy Behind the Life quality Index and Empirical Verification, Working material of JCSS, 2005.

[17] Stewart M.S. \& Melchers R.E. (1997) Probabilistic risk assessment of engineering system. Chapman \& Hall, London, 1997, 274 p.

[18] Trbojevic V. M. (2003) Development of Risk Criteria for Road Tunnels. $5^{\text {th }}$ International Conference - Safety in Road and Rail Tunnels. Marseille, 2003. 
[19] Vrouwenvelder A., Holický M., Tanner C.P., Lovegrove D.R., Canisius E.G. (2001) CIB Report. Publication 259. Risk assessment and risk communication in civil engineering. CIB, 2001.

[20] Vrouwenvelder A.C.W.M. \& Krom A.H.M. (2004) Hazard and the Consequences for Tunnels Structures and Human Life. 1st International Symposium Safe and Reliable Tunnels in Prague, CUR, Gouda, The Netherlands, 2004.

[21] Weger D. de, M.M. Kruiskamp and J. Hoeksma (2001). Road Tunnel Risk Assessment in the Netherlands - TUNprim: A Spreadsheet Model for the Calculation of the Risks in Road Tunnels. ESREL 2001.

[22] Worm, E.W. and Hoeskma, J. (1998) The Westerschelde Tunnel: Development and application of an integrated safety philosophy, Safety in Road and Rail Tunnels, 3rd International Conference organised by University of Dundee and ITC Ltd., Nice, France, 1998. 